\title{
Communication \\ Millimeter-wave New Radio Test Zone Validation for MIMO Over-the-air Testing
}

\author{
Fengchun Zhang, Lassi Hentilä, Pekka Kyösti and Wei Fan
}

\begin{abstract}
Standardization efforts to define radiated metrics and test methods for verifying millimeter-wave (mmWave) new radio (NR) devices are currently ongoing. Multi-probe anechoic chamber (MPAC) method, which is capable of reproducing spatial fading channels in the test zone, is selected as the reference solution for NR device testing. It is of importance to ensure that the defined testing propagation environment in the test zone is correctly implemented. In this paper, a novel algorithm is proposed to determine the wideband power-angle-delay profile of the reconstructed channels in the test zone for mmWave NR. The proposed algorithm is shown to be generic (applicable for both 2D and 3D testing setup), applicable for both near-field and far-field conditions and robust (i.e. insensitive to measurement distance errors).
\end{abstract}

Index Terms-MIMO over-the-air testing, radio channel modeling, channel model validation, wideband power-angle-delay profile, millimeter-wave communication

\section{INTRODUCTION}

Performance testing of $5 \mathrm{G}$ radios, including both radio and baseband parts, is a mandatory step in product design and development [1], [2]. Traditional cabled test methods, where testing signals are guided to reserved antenna ports on the device under test (DUT) via radio frequency (RF) cables, have become obsolete for $5 \mathrm{G}$ radios operating at frequency range (FR) 2 (i.e. from $24.25 \mathrm{GHz}$ to $52.6 \mathrm{GHz}$ ), due to highly integrated system designs [2]-[4]. It is expected that $5 \mathrm{G}$ radio testing will move exclusively to radiated over-theair (OTA) modes, where DUT internal antennas are served as direct interfaces to testing signals. Valid test propagation environment is important to benchmark the performance of $5 \mathrm{G}$ new radio (NR) equipped with various types of antenna and chipset designs. Standardized MIMO OTA testing is essential for protocol functional testing, verification of advanced baseband functionality in NR (e.g. carrier aggregation, massive MIMO and beam forming), and radio resource management (RRM) including handover and throughput testing. Significant efforts are ongoing in standardization to define radiated metrics and test methodology for verifying multi-antenna reception performance of 5G NR user equipment (UE) operating in FR1 and FR2 [5]. The simplified three dimensional (3D) multiprobe anechoic chamber (MPAC) test method is selected as the reference testing methodology for FR2 NR MIMO OTA testing [5].

Fengchun Zhang and Wei Fan are with Antenna Propagation and Millimeterwave Systems (APMS) section at Department of Electronic Systems, Aalborg University, Denmark. (Corresponding author: Wei Fan. Email: wfa@es.aau.dk)

Lassi Hentilä and Pekka Kyösti is with Keysight Technologies Finland oy, Finland.
In order to ensure that the defined testing propagation environment is correctly and accurately reproduced, test zone channel validation is mandatory. The purpose of test zone validation is to check whether both the physical test setup (probes, cables, anechoic chamber, and etc) and the channel model implemented by the fading channel emulator are correct and accurate. Channel validation measurements in the 2D uniform MPAC setup for 4G MIMO terminals are widely reported in the academia and industry, see e.g. [6]-[10]. The focus has been on validating whether various channel parameters (i.e. fading distribution, power-delay profile (PDP), power-Doppler profile, cross-polarization ratio and spatial profile) of the emulated channels inside the test zone are accurately implemented via comparing with those of the target channels. Validation of channel spatial profile in the test zone is a challenging task. Spatial correlation is used to characterize the channel spatial profile for 4G MIMO OTA testing, partly due to the importance of correlation in multi-antenna performance (i.e. spatial multiplexing and diversity). It was pointed out in [3], [9], [11] that power angle spectrum (PAS) of the emulated channels are more relevant for 5G NR in FR2. Furthermore, PAS indicates directly where the signal originates, which is more relavant for beam-steerable DUTs. Therefore, PAS based metric, PAS similarity percentage (PSP) is discussed for FR2 DUT in standardization [5].

It is essential that channel validation measurements should be performed in a fast, accurate and convenient manner. However, the task is not trivial for FR2 systems, due to several unsolved technical difficulties in the literature:

- 2D channel models and 2D uniform MPAC setups were defined for 4G MIMO OTA testing, and hence virtual uniform linear array (ULA) [6], [10] and uniform circular array (UCA) [9] were used to sample the test zone. Such virtual array configurations, however, are not sufficient for channel validation in 3D MPAC setups.

- For cost-saving purpose, the anechoic chamber should be compact, while the test zone size should be sufficiently large to encompass the DUT. The far field condition, which holds true for $4 \mathrm{G}$ MIMO OTA testing, will be violated for FR2 testing.

- A much larger number of spatial locations to cover the test zone is required at FR2 due to its much smaller wavelength and necessity of 3D virtual arrays, which would in turn result in long measurement time.

These challenges would become even more demanding for base station (BS)-type DUT at FR2. In this paper, a novel algorithm is proposed to validate the wideband power-angle-delay profile 
(PADP) of emulated spatial channels in the test area. The proposed algorithm differs from state-of-art works, since it is generic (i.e. applicable for both 2D and 3D MPAC setups with arbitrary measurement distance), fast (which only requires a few measured spatial locations) and computationally efficient (i.e. search complexity significantly reduced). Our proposed solution is essentially an extension and improvement of the reference method presented in [9], which was only applicable when far-field condition is met.

\section{Problem statement and Proposed Method}

\section{A. Problem Statement}

An illustration of the 3D MPAC testing system is shown in Fig. 1, where OTA antennas are arranged around the DUT. Each OTA antenna port is connected to a mmWave channel emulator (CE) interface port. The objective is to emulate realistic multipath channel conditions in the test zone with the help of the multiple OTA antennas and the CE. 3D geometrybased stochastic channel (GBSC) models are selected as the reference multipath channels for performance testing [5], [12]. The popular prefaded signal synthesis (PFS) channel emulation technique [3], [11] is implemented to map radio channel models onto the OTA antennas (i.e. channel impulse responses (CIRs) implemented in the $\mathrm{CE}$ ). The PFS technique aims to reconstruct a spatial channel model statistically similar to the reference channel. That is, same channel parameters, including PDP, power Doppler spectrum, Ricean K-factor, amplitude fading distribution, cross-polarization power ratio, and PAS, etc are reconstructed inside the test zone.

For FR2 NR UE testing, the number of OTA antennas is $K=6$ and the OTA antenna configuration is detailed in Table 6.2.3-1 in [5]. The radius of the OTA antenna sphere and test zone sphere is $R$ and $r$, respectively. As specified in [5], the test zone radius is $0.05 \mathrm{~m}$, while the MPAC sphere radius can be as small as $0.75 \mathrm{~m}$. Therefore, the far field assumption $R \geq \frac{2(2 r)^{2}}{\lambda}$ is not met for $5 \mathrm{G} \mathrm{NR}$ at FR2. It should be noted that a test zone with a radius of $0.05 \mathrm{~m}$ is small if the DUT is viewed as a black box. Generally speaking, the exact antenna size of the DUT is unknown since the device will be in its own casing during the test and this also depends on other factors such as ground coupling effects that depend on the design. The largest device size (e.g. diagonal) could be used; however, this would lead to unnecessarily demanding requirement on chamber size. It might be sufficient from practical point of view since the physical size of mmWave antenna systems will be rather small (e.g. $5 \mathrm{~cm}$ corresponding to $4.7 \lambda$ at $28 \mathrm{GHz}$ ), and the radiating area of the DUT is limited as well. Furthermore, if a larger test zone is needed due to the increased DUT size, the virtual array adopted in this paper can be easily extended to cover a larger test zone via allocating more virtual array elements along the enlarged circles to ensure a $0.5 \lambda$ element spacing along each circle. If the probe locations in a MPAC setup are kept unchanged, a larger test zone size makes the near field problem more pronounced, which further motivates our work.

For channel validation purposes, a vector network analyzer (VNA) is utilized to record the channel frequency response

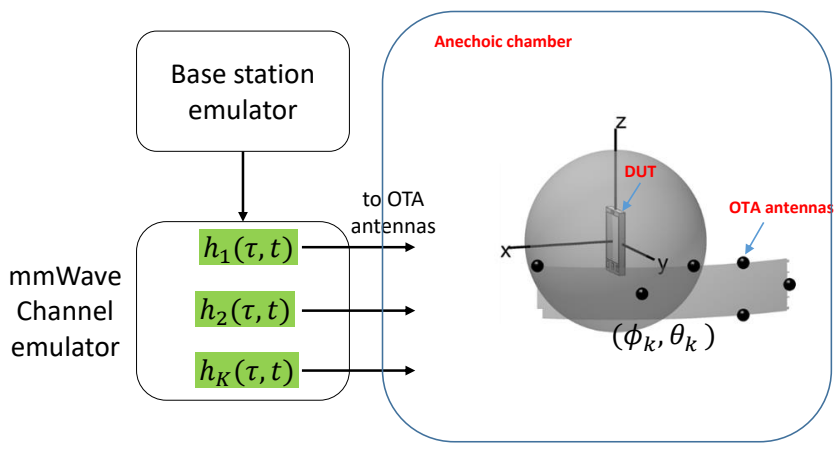

Fig. 1. System diagram for the 3D MPAC setup for FR2 NR performance testing. $h_{q}(\tau, t)$ is the wideband time-variant CIRs loaded for the $q$-th OTA antenna, with $q \in[1, Q]$.

(CFR), and the CIR can be obtained via inverse discrete Fourier transform (IDFT). Note the VNA is too slow to record real-time time-variant channels (continuous mode in the CE) at FR2. Fortunately, the CIR profiles can be paused and stepped in channel snapshots in the CE for validation purposes. Therefore, the time-variant profiles can hence be obtained via performing many "static" measurements. Note that for channel spatial profile measurement, only the vertical polarization is required, due to the fact that same procedure can be repeated for the other polarization.

Channel spatial profile measurement has been a popular research topic. Directional channel scanning, where a directive antenna is steered with the help of a turntable to record the channel spatial profiles, has been the dominant solution at mmWave frequency bands in the literature [13]-[16]. This is mainly due to its high gain, relatively low cost and simplicity. The directional channel scanning scheme can be used for test zone channel validation in principle. However, there are some practical problems. First, we should rotate the horn antenna both in azimuth and elevation to cover the angle sector of interest, which could be time-consuming. Second, the horn antenna should be directive enough such that we can distinguish signals radiated from each OTA antenna. This essentially also implies much longer measurement time. Also, the aperture size of the horn antenna is typically large, making it difficult to point to the target OTA antenna, especially when the OTA probe antennas are closely spaced. In addition, the impinging angles of the emulated channels cannot be accurately estimated with the horn antenna. Furthermore, radiated signals from all probe antennas should be measured simultaneously in the validation measurement, since this is the case for the actual throughput measurement. A virtual array solution, where a reference omnidirectional antenna is placed in many predefined spatial locations [17], is an attractive alternative for this validation purpose since it provides insights into how the signals radiate from the multi-probe antennas, and possible reflections inside the anechoic chamber. A virtual array solution is capable of estimating the PADPs of the channels emulated by the simultaneously radiating probe antennas. Although a virtual array solution only works for static channel scenarios in principle, it is applicable for time-variant channels in the MIMO OTA channel validation since the emulated channels 
is controllable and repeatable (i.e. CIRs can be stepped and paused, and replayed for different spatial locations). A virtual ULA is a conventional choice to record spatial correlation for the 2D MPAC setup. However, a ULA is not suitable for measuring channel spatial profiles in the 3D MPAC setups. In [9], a virtual UCA is formed in the test zone to estimate the wide-band PADP of the emulated channels. However, the algorithm was only applicable to the 2D MPAC setup when far field condition is met.

Assuming $N$ channel snapshots were stepped in the CE, $L$ frequency samples were recorded per channel snapshot in the VNA, and we have $M$ spatial samples that compose a virtual array. The size of the recorded data is $N \times M \times L$. The total measurement time mainly depends on the selection of $N, M$ and $L$. The selection of $M$ is a compromise between measurement time and accuracy. The virtual array should be large enough to compass the DUT, and the spacing between virtual array elements should not exceed half wavelength to avoid spatial aliasing. On the other hand, a small $M$ is required to reduce measurement time. Therefore, the objective of the work is to design a virtual array with few elements and nearfield array algorithm that is able to determine wideband PADP of the emulated channels in the test zone in the 3D MPAC setup.

In this paper, a 3D virtual antenna array is realized by successively positioning a reference omnidirectional antenna at predefined locations. For each location, the CFRs of many fading channel snapshots are recorded. Once the CFRs of all locations, i.e. all virtual array elements, have been measured, a proper array processing algorithm should be applied to extract the channel characteristics.

\section{B. Proposed method}

1) Virtual array formulation: A 3D virtual array configuration is required to detect $3 \mathrm{D}$ spatial profiles of the emulated channels in the 3D MPAC system. For a given 3D MPAC setup to emulate a specified spatial channel model (with specified directions of arrival and a given power range of channels), a virtual antenna array can be optimized to have non-uniform element spacings and a minimum number of antenna elements. However, this 'customized' array might not work well for other channel models. Therefore, a more generic virtual array should be employed to validate a variaty of channel models in the test zone. In addition, it is important that the virtual array configuration should be easily supported by mechanical turntable or linear slider, which exists in practical anechoic chambers, to facilitate automated measurements. Furthermore, the number of virtual array elements should be limited to reduce the measurement time.

A comprehensive 3D array configuration (e.g. a sphere array or a cubic array) can offer good performance, yet impractical due to its long measurement time. In this work, a virtual array composed of three semi-circle arrays, one on the horizontal plane and two parallel on the vertical plane, is considered, as shown in Fig. 2. This virtual array structure can be easily realized in the practical setup as explained and it is a generic configuration for arbitrary 3D MPAC setups. The array structure

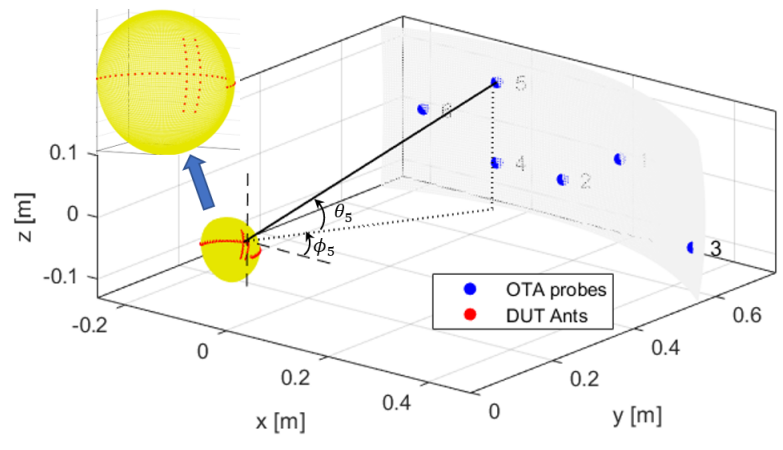

Fig. 2. An illustration of the virtual array configuration and 3D MPAC setups.

requires significantly less array elements compared to full array structure, yet it offers 3D spatial profile estimation capability. Note that the origin of the coordinate system is the center of both the OTA antenna sphere and the test zone sphere. The elevation angle $\theta$ and azimuth angle $\phi$ are defined in Fig. 2.

2) Near-field sequential search algorithm: As detailed in [9], a two-stage sequential search was proposed to reduce the computation complexity associated with angle and delay domain joint search. The same sequential search strategy yet re-designed for the near field condition is applied in this work, as discussed below.

The received signal vector by $M$ DUT antennas at the $n$-th channel snapshot, ignoring the noise, is given as:

$$
\boldsymbol{y}^{n}(f)=\mathbf{A}(f) \cdot \mathbf{s}^{n}(f), \boldsymbol{y} \in \mathbb{C}^{M \times 1},
$$

where $f$ is the operating frequency, $\mathbf{s} \in \mathbb{C}^{Q \times 1}$ denotes the transmitted signal vector from the $Q$ OTA probe antennas and $\mathbf{A} \in \mathbb{C}^{M \times Q}$ represents the channel transfer function from the OTA probe antennas to the DUT antennas. The signal transmitted from the $q$-th OTA probe antenna at the $n$-th snapshot is written as:

$$
s_{q}^{n}(f)=\sum_{i=1}^{p_{q}} \alpha_{q, i}^{n} e^{-j 2 \pi f \tau_{q, i}},
$$

where $\alpha_{q, i}^{n}$ denotes the complex amplitude of the $i$-th path in the $q$-th direction at the $n$-th snapshot, $\tau_{q, i}$ is the delay of the $i$ th path in the $q$-th direction, and $p_{q}$ represents the total number of paths in the $q$-th direction. The channel transfer function between the $m$-th DUT antenna and the $q$-th OTA probe antenna, i.e. the $(m, q)$-th entry of $\mathbf{A}(f)$, can be expressed by:

$$
a_{m, q}(f)=\frac{c}{4 \pi f d_{m, q}} e^{-\frac{j 2 \pi f}{c} d_{m, q}}
$$

where $c$ is the speed of light and $d_{m, q}=\left|\vec{r}_{m}-\vec{R}\left(\theta_{q}, \phi_{q}\right)\right|$ denotes the distance between the $m$-th DUT antenna and the $q$-th OTA probe antenna with $\vec{r}_{m}$ and $\vec{R}\left(\theta_{q}, \phi_{q}\right)$ representing the respective location vectors. The location vector for the probe antenna in direction $(\theta, \phi)$ can be written as:

$$
\vec{R}(\theta, \phi)=R \cdot \vec{k}(\theta, \phi),
$$

where $R$ represents the radius of the OTA probe antenna sphere and $\vec{k}(\theta, \phi)$ denotes the unit location vector, which is expressed as:

$$
\vec{k}(\theta, \phi)=[\cos \theta \cos \phi ; \cos \theta \sin \phi ; \sin \theta] .
$$


Under the far-field assumption, we have $R \gg r$. Applying Taylor series expansion, the distance term $\left|\vec{r}_{m}-\vec{R}\left(\theta_{q}, \phi_{q}\right)\right|$ can be thereby simplified as

$$
\left|\vec{r}_{m}-\vec{R}\left(\theta_{q}, \phi_{q}\right)\right|=R-\vec{r}_{m}^{T} \cdot \vec{k}\left(\theta_{q}, \phi_{q}\right),
$$

where $(\cdot)^{T}$ denotes the transpose operator. Substituting (6) back into (3) and removing the common phase term and path loss term introduced by $R, a_{m, q}(f)$ in (3) is simplified to be the far-field array factor of the DUT array, which is given as:

$$
\underline{a}_{m, q}(f)=e^{\frac{j 2 \pi f}{c}} \vec{r}_{m}^{T} \cdot \vec{k}\left(\theta_{q}, \phi_{q}\right) .
$$

Note that $a_{m, q}(f)$ in (3) represents the near-field array factor of the DUT array.

Below, a sequential 1D search method is discussed: in step 1 , the angles of arrival are estimated by applying multiple signal classification (MUSIC) algorithm; in step 2, the PDPs are obtained based on the angles of arrival estimated in step 1.

The corariance matrix of $\boldsymbol{y}$ can be calculated as:

$$
\mathbf{R}_{y}=\sum_{n} \sum_{f} \boldsymbol{y}^{n}(f)\left[\boldsymbol{y}^{n}(f)\right]^{H}, \mathbf{R}_{y} \in \mathbb{C}^{M \times M},
$$

where $(\cdot)^{H}$ denotes the complex conjugate transpose operator. By applying MUSIC algorithm, the PAS can be obtained as

$$
P(\theta, \phi)=\frac{1}{\boldsymbol{\omega}(\theta, \phi) \mathbf{V} \mathbf{V}^{H} \boldsymbol{\omega}^{H}(\theta, \phi)},
$$

where $\boldsymbol{\omega}(\theta, \phi) \in \mathbb{C}^{1 \times M}$ is the weight vector of the DUT array and $\mathbf{V}$ is the noise-subspace matrix obtained from eigendecomposition of $\mathbf{R}_{y}$. The far-field MUSIC is implemented, when we select $\boldsymbol{\omega}(\theta, \phi)$ according to the far-field array factor in (7) as:

$$
\underline{\omega}_{m}(\theta, \phi)=e^{\frac{-j 2 \pi f_{c}}{c}} \vec{r}_{m}^{T} \cdot \vec{k}(\theta, \phi),
$$

where $f_{c}$ denotes the center frequency. Similarly, the near-field MUSIC is implemented, when we select $\boldsymbol{\omega}(\theta, \phi)$ according to the near-field array factor in (3) as:

$$
\omega_{m}(\theta, \phi)=\frac{c}{4 \pi f_{c}\left|\vec{r}_{m}-\vec{R}(\theta, \phi)\right|} e^{\frac{j 2 \pi f_{c}}{c}\left|\vec{r}_{m}-\vec{R}(\theta, \phi)\right|} .
$$

The directions of arrival $\left(\hat{\theta}_{q}, \hat{\phi}_{q}\right)$ can be estimated via finding the peaks in the power spectra $P(\theta, \phi)$ in (9), based on which, the delay profile for each direction can be extracted. To reduce the interferences introduced by the other directions in the PDP of the target direction, the PDP can be obtained via setting the array to steer a beam to the target direction $\left(\hat{\theta}_{q}, \hat{\phi}_{q}\right)$ and meanwhile locate the nulls towards the interfering directions, i.e. $\left(\hat{\theta}_{i}, \hat{\phi}_{i}\right)$ for $i \in[1, Q]$ and $i \neq q$. To accomplish it, a proper complex weight vector $\varpi\left(\hat{\theta}_{q}, \hat{\phi}_{q}\right) \in \mathbb{C}^{M \times 1}$ should be implemented at DUT elelments, which is given as [18]:

$$
\varpi\left(\hat{\theta}_{q}, \hat{\phi}_{q}\right)=\left[\mathbf{I}_{M}-\boldsymbol{\Omega}\left(\boldsymbol{\Omega}^{H} \boldsymbol{\Omega}\right)^{-1} \boldsymbol{\Omega}^{H}\right] \boldsymbol{\omega}^{T}\left(\hat{\theta}_{q}, \hat{\phi}_{q}\right),
$$

where $\mathbf{I}_{M}$ is the identity matrix of size $M$ and $\Omega \in \mathbb{C}^{M \times(Q-1)}$ is the nulling matrix with its $i$-th column vector equal to $\boldsymbol{\omega}^{T}\left(\hat{\theta}_{i}, \hat{\phi}_{i}\right)$ for $i \in[1, Q]$ and $i \neq q$.

The CIR in direction $\left(\hat{\theta}_{q}, \hat{\phi}_{q}\right)$ can be estimated as:

$$
\hat{s}_{q}^{n}(\tau)=\left[\varpi^{H}\left(\hat{\theta}_{q}, \hat{\phi}_{q}\right) \varpi\left(\hat{\theta}_{q}, \hat{\phi}_{q}\right)\right]^{-1} \varpi^{T}\left(\hat{\theta}_{q}, \hat{\phi}_{q}\right) \boldsymbol{y}^{n}(\tau),
$$

where $\boldsymbol{y}^{n}(\tau)$ is obtained via IDFT of $\boldsymbol{y}^{n}(f)$. The PDP in direction $\left(\hat{\theta}_{q}, \hat{\phi}_{q}\right)$ is given by

$$
P\left(\hat{\theta}_{q}, \hat{\phi}_{q}, \tau\right)=\sum_{n}\left|\hat{s}_{q}^{n}(\tau)\right|^{2} .
$$

The peaks in the PDP give the power and delay estimates for $i \in\left[1, p_{q}\right]$. The marginal profiles, i.e. the PDP and PAS, can be easily derived from the joint profiles. The PSP can also be obtained based on the PAS and the DUT configurations [11].

There are several publications on wide-band joint PADP estimation in the state-of-art works, e.g. the well-known joint angle-delay estimation (JADE) algorithm [19]. The JADE algorithm suffers from the high computational complexity mainly introduced by the eigen decomposition of the large dimension correlation matrix $\mathbf{R} \in \mathbb{C}^{M L \times M L}$ and 3D bruteforce search in the elevation angle, azimuth angle and delay domains. In contrast, the computation complexity of the proposed algorithm based on the two-stage sequential search method can be significantly reduced, where the eigen decomposition is based on the correlation matrix $\mathbf{R} \in \mathbb{C}^{M \times M}$ and the bruteforce search space shrinks to $2 \mathrm{D}$, i.e. the elevation angle and azimuth angle domains.

Also, it is possible to estimate the joint PADP per channel snapshot using conventional channel estimation algorithms, e.g. wideband beamforming algorithm, SAGE algorithm, and maximum likelihood algorithm [20], [21], and then perform the average over channel snapshots afterwards to obtain the wideband PADP. However, the algorithm complexity is generally very high due to the time-consuming JADE for each channel snapshot is needed. In the MIMO OTA channel validation standards [7], the number of snapshots for the fading channel is 20000 or at least 2000 in the channel validation stage.

Therefore, the proposed algorithm based on the two-stage sequential search offers low computation cost, comparing with the state-of-art algorithms.

\section{Simulation Results}

In this section, the proposed algorithm is numerically validated. One representative 3GPP cluster delay line (CDL) spatial channel model for indoor office $(\mathrm{InO})$ scenario, i.e. InO-CDL-A in Table 7.2.2-6 in [5] is selected as the reference channel. Note that channel modeling features, e.g. channel scaling, including of BS antenna arrays and beams to channel models and removing unwanted randomness of the channel model realization are considered according to [5], [12]. The CIRs in the CE are generated using Keysight GCM simulation tool.

The virtual array is composed of 51 elements (i.e. 31 elements on the horizontal circle to cover $\left[-90^{\circ}, 90^{\circ}\right]$ and 10 elements on each vertical circle to cover $\left[-30^{\circ}, 30^{\circ}\right]$ ). The element spacing on all semi-circles is set to $0.5 \lambda$. The simulation frequency and bandwidth is set to $28 \mathrm{GHz}$ and 200 $\mathrm{MHz}$ respectively. The vertical sectors of the measurement array are limited to $60^{\circ}$ and the horizontal sector to $180^{\circ}$ with the boresight direction pointing towards the OTA antennas, since the OTA antennas are concentrated in the horizontal plane, as shown in Fig. 1. The measurement antenna should 


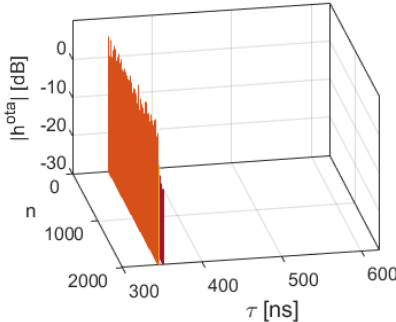

(a)

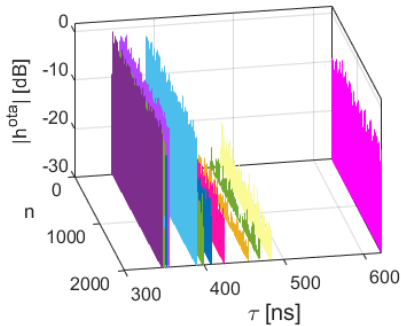

(c)

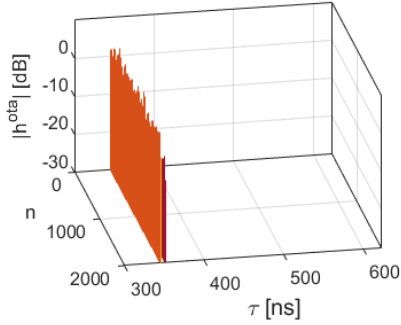

(e)

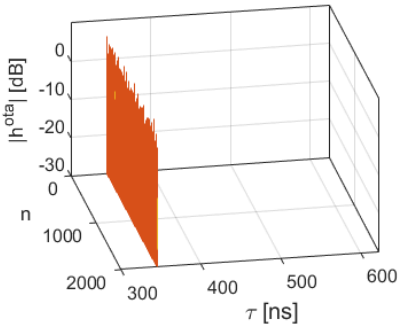

(b)

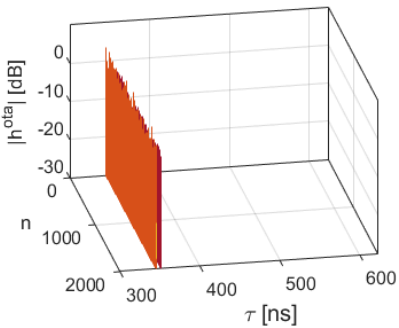

(d)

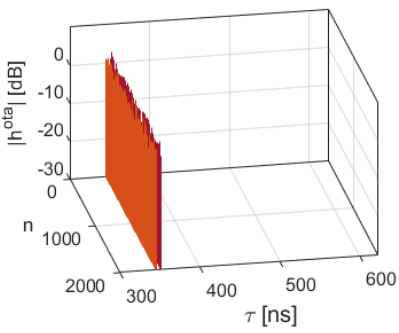

(f)
Fig. 3. Time-variant CIRs radiated from the 6 OTA antennas in (a) to (f), respectively .

be isotropic in principle. In practice it is sufficient to have omni-directional pattern in azimuth and a wide beam width in elevation, e.g., Bi-cone antennas. The accuracy for the elevation domain would be affected if the measurement antenna has a very narrow beam in the elevation domain. Isotropic antennas are assumed for the virtual array elements in this work.

The time-variant CIRs radiated from the six OTA antennas are shown in Fig. 3. The InO-CDL-A channel model consists of 23 paths with a maximum excess delay around $290 \mathrm{~ns}$. As shown in Fig. 3, only path 1 (with a delay around $340 \mathrm{~ns}$ ) is dominant in most OTA probes. This is due to the BS array filtering effect introduced in [5], where the BS antenna only steers a beam towards the strongest path in each channel model.

We firstly apply the MUSIC algorithm in (9) with the weight vector in (10) for the far-field case and with the weight vector in (11) for the near-field case to determine the 3D pseudo PAS. As shown in Fig. 4 (a), with the algorithm in [9], not all six paths can be identified due to the low resolution in the elevation domain and the strong ripples (only $6 \mathrm{~dB}$ below the strongest path) in the PAS, introduced by the near-field effects. With the proposed near-field MUSIC algorithm, all six paths can be clearly identified due to the sharp peaks and the weak ripples (more than $62 \mathrm{~dB}$ below the highest peak) in the PAS, and their angle estimates match perfectly with the target values shown in Fig. 4 (b). The proposed virtual array structure offers good angle resolution both in azimuth and elevation domains.

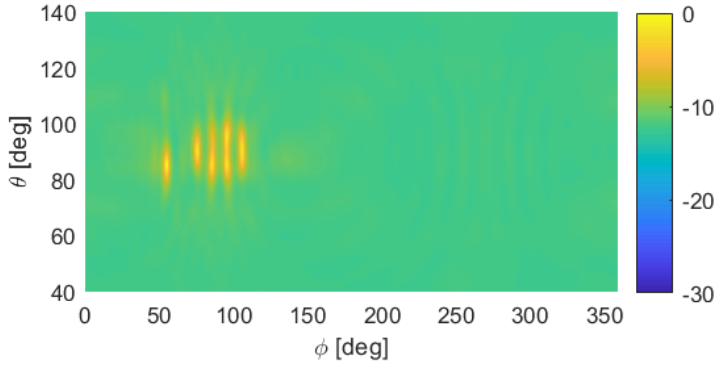

(a)

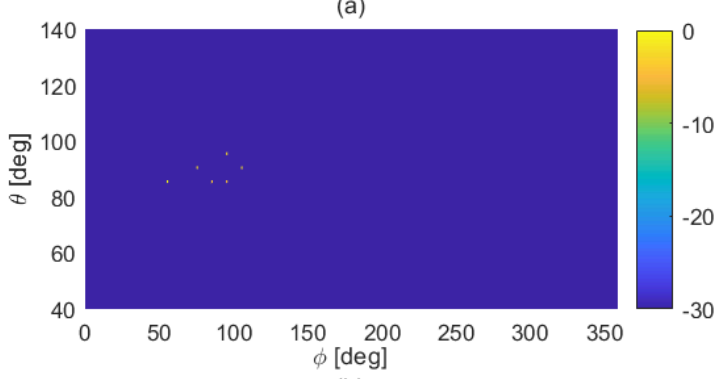

(b)

Fig. 4. PAS estimated with the far-field MUSIC (a) and near-field MUSIC algorithm (b).

After extracting the angular locations of the active OTA antennas, the next step is to estimate the PDP associated with each OTA antenna. As explained, the virtual array can steer the beam to each OTA path direction to obtain the corresponding PDP, as shown in Fig. 5. It can be observed that via applying beamforming without nulling operation, many "fake" paths might appear in the resulted PDPs (blue dashed lines), besides the target path, which lead to inaccurate validation results. As discussed previously, these "fake" paths (i.e. the interferences of other paths) can be greatly eliminated via setting the virtual array to steer a beam towards the target path direction while to form nulls towards all other path directions, as illustrated by the black dashed lines. With the nulling operation, the estimated PDP matches excellently with the target profile for each OTA antenna. Therefore, the nulling operation is essential to obtain the true delay estimates in target directions. The power per path for each antenna can be obtained from the resulted PDP. The total power per OTA antenna can then be calculated. The estimated total power per probe using the proposed algorithm matches excellently with the target value, with a deviation smaller than $0.1 \mathrm{~dB}$.

In a practical test setup, the measurement distance $R$ can be measured approximately (e.g. using a measuring tape or a laser) before the validation process. Due to the measurement error, the measured $R$ might slightly deviate from the actual $R_{o}$. To investigate the sensitiveness of the estimation algorithm to the errors in $R$, the error metrics such as the power level of the ripples in PAS, angle estimation error, delay estimation error and the power error of the OTA probes are introduced. In the simulation, the setup is kept unchanged, except that the measured $R=0.75 \mathrm{~m}$ is used for channel estimation in equations (9) to (14) and the actual $R_{o}=R+\Delta R$ with $\Delta R \in$ $[-0.05,0.05] \mathrm{m}$ is set for channel generation in equation (1). As discussed previously, many ripples exist besides the dominant peaks in Fig. 4(b). The simulation results show that the ripple 


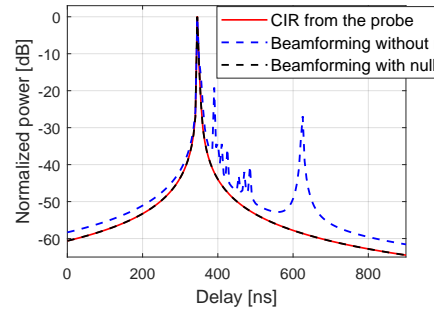

(a)

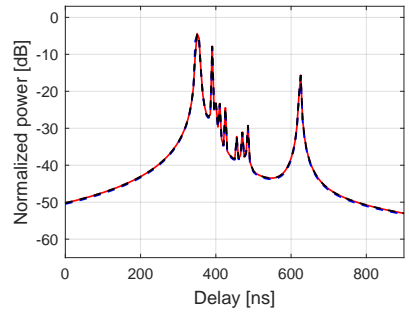

(c)

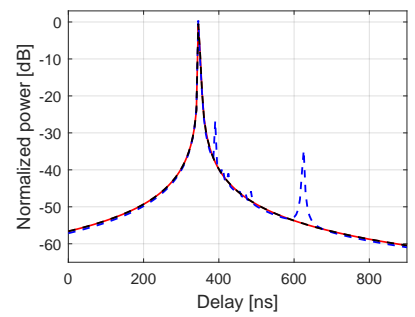

(e)

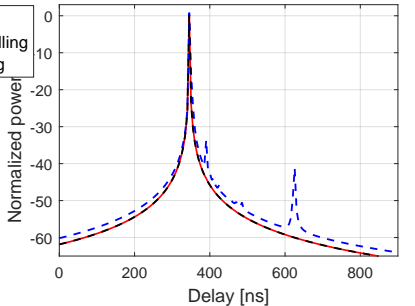

(b)

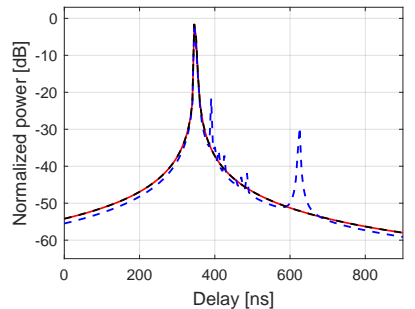

(d)

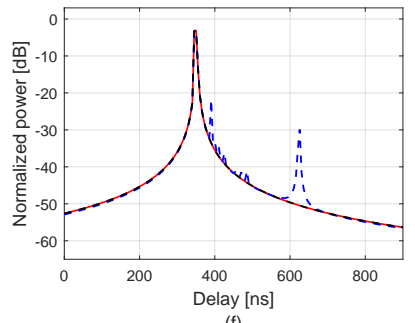

Fig. 5. The PDPs in the directions of the 6 OTA antennas, plotted in (a) to (f), respectively.

level in the PAS rises as the $|\Delta R|$ increases, implying that some fake peaks might appear when the distance error keeps increasing. Furthermore, the distance error within $[-0.05,0.05]$ $\mathrm{m}$ has a neglectable effect on estimation accuracy of angle, delay and power value per OTA probe antenna, demonstrating that the proposed DUT array and algorithm is not sensitive to the distance errors. It is worth noting that a measurement error less than $5 \mathrm{~cm}$ in $R$ can generally be achieved in practice. Hence, the proposed estimation algorithm is applicable in a practical testing.

\section{CONCLUSION}

The MPAC method is a promising solution for testing 5G antenna systems over-the-air, which aims to physically emulate spatial channel environments inside the test zone to approximate the realistic RF environments experienced by the DUT. Estimation of emulated spatial channels in the test zone is essential to ensure that the defined testing channel environments are correctly reproduced. In this paper, a novel channel estimation algorithm is proposed to estimate the emulated wideband channels within the test zone at mmWave frequencies. The proposed algorithm is a generic channel estimation algorithm which works for 3D channel models under the more challenging near-field conditions. The simulation results demonstrate that the proposed algorithm can accurately estimate the reconstructed channels inside the test zone even in the present of errors in measurement distance $R$. Therefore, this algorithm is applicable in a practical MPAC testing setup.

\section{REFERENCES}

[1] Y. Qi, G. Yang, L. Liu, J. Fan, A. Orlandi, H. Kong, W. Yu, and Z. Yang, " $5 \mathrm{~g}$ over-the-air measurement challenges: Overview," IEEE Transactions on Electromagnetic Compatibility, vol. 59, no. 6, pp. 1661-1670, Dec 2017.

[2] M. Rumney, "Testing 5g: Time to throw away the cables," Microw. J., vol. 59, no. 11, 2016

[3] W. Fan, P. Kyosti, M. Rumney, X. Chen, and G. F. Pedersen, "Overthe-air radiated testing of millimeter-wave beam-steerable devices in a cost-effective measurement setup," IEEE Communications Magazine, vol. 56, no. 7, pp. 64-71, July 2018.

[4] W. Hong, "Solving the 5g mobile antenna puzzle: Assessing future directions for the $5 \mathrm{~g}$ mobile antenna paradigm shift," IEEE Microwave Magazine, vol. 18, no. 7, pp. 86-102, Nov 2017.

[5] 3GPP, "Study on radiated metrics and test methodology for the verification of multi-antenna reception performance of nr user equipment (ue);", June 2020.

[6] C. T. Plan, " $2 \times 2$ downlink mimo and transmit diversity over-the-air performance version 1.2," 2018.

[7] G. TR37.976, "Measurement of radiated performance for multiple input multiple output (mimo) and multi-antenna reception for high speed packet access (hspa) and lte terminals release 13," 2016.

[8] Lin Guo, Can Sun, Xudong An, Xiao Zhang, and Meng Yang, "Over the air mimo channel model validation," in 2013 7th European Conference on Antennas and Propagation (EuCAP), 2013, pp. 1848-1852.

[9] W. Fan, F. Zhang, and Z. Wang, "Over-the-air testing of $5 \mathrm{~g}$ communication systems: Validation of the test environment in simple-sectored multiprobe anechoic chamber setups," IEEE Antennas and Propagation Magazine, pp. 0-0, 2019.

[10] A. Scannavini, L. J. Foged, N. Gross, L. Hentila, J. Virtala, and K. Raju, "Test zone characterization for the multiprobe anechoic chamber setup (mpac)," in 2016 10th European Conference on Antennas and Propagation (EuCAP), 2016, pp. 1-5.

[11] P. Kyösti, L. Hentilä, W. Fan, J. Lehtomäki, and M. Latva-Aho, "On radiated performance evaluation of massive mimo devices in multiprobe anechoic chamber ota setups," IEEE Transactions on Antennas and Propagation, vol. 66, no. 10, pp. 5485-5497, 2018.

[12] G. 38.901, "Study on channel model for frequencies from 0.5 to 100 ghz," January 2020.

[13] T. S. Rappaport, S. Sun, R. Mayzus, H. Zhao, Y. Azar, K. Wang, G. N. Wong, J. K. Schulz, M. Samimi, and F. Gutierrez, "Millimeter wave mobile communications for $5 \mathrm{~g}$ cellular: It will work!" IEEE Access, vol. 1, pp. 335-349, 2013.

[14] J. Vehmas, J. Jarvelainen, S. L. H. Nguyen, R. Naderpour, and K. Haneda, "Millimeter-wave channel characterization at helsinki airport in the 15, 28, and $60 \mathrm{ghz}$ bands," in 2016 IEEE 84th Vehicular Technology Conference (VTC-Fall). IEEE, 2016, pp. 1-5.

[15] S. Collogne, G. Zaharia, and G. El Zein, "Experimental investigation of the spatial and temporal characteristics of the $60 \mathrm{ghz}$ radio propagation within residential environments," in Signals, Circuits and Systems, 2003. SCS 2003. International Symposium on, vol. 2. IEEE, 2003, pp. 417-420.

[16] R. Müller, R. Herrmann, D. A. Dupleich, C. Schneider, and R. S Thomä, "Ultrawideband multichannel sounding for mm-wave," in The 8th European Conference on Antennas and Propagation (EuCAP 2014). IEEE, 2014, pp. 817-821.

[17] J. Medbo, N. Seifi, and H. Asplund, "Frequency dependency of measured highly resolved directional propagation channel characteristics," in European Wireless 2016; 22th European Wireless Conference, 2016, pp. $1-6$.

[18] H. Trees, "Optimum array processing (detection, estimation, and modulation theory, part iv) wiley," 2002.

[19] A.-J. Van Der Veen, M. C. Vanderveen, and A. J. Paulraj, "Joint angle and delay estimation using shift-invariance properties," IEEE Signal Processing Letters, vol. 4, no. 5, pp. 142-145, 1997.

[20] F. Zhang and W. Fan, "Near-field ultra-wideband mmwave channel characterization using successive cancellation beamspace uca algorithm," IEEE Transactions on Vehicular Technology, vol. 68, no. 8, pp. 72487259, 2019.

[21] Y. Ji, W. Fan, and G. F. Pedersen, "Channel characterization for wideband large-scale antenna systems based on a low-complexity maximum likelihood estimator," IEEE Transactions on Wireless Communications, vol. 17, no. 9, pp. 6018-6028, 2018. 